pendent of the special interests of students. In other words, it is important for all biological students, not just for potential taxonomists.

little attempt has been made in Britain, largely due to the departmental system, to teach systematics as a unified subject. Thus it is possible for the same topics (classifica. tion, phylogeny, species concepts, evolution, etc.) to be taught separately and in widely diverse (sometimes even conflicting) ways in different departments within the same biological school. Wherever possible, taxonomy should be taught as a unified subject and not compartmentalized between botany, palæontology, zoology, etc.

The teaching of taxonomy in Great Britain could often be greatly improved. In many universities it is not taught at all in a recognizable form. Undergraduate teaching in taxonomy should enable the student to negotiate with reasonable success almost any group of organisms without being a specialist in them, and with a minimum of help from specialists. To achieve this it is necessary to instil the basic principles of taxonomy which are applicable, with some modification, to all groups of organisms. At the same timo, teaching should not be restricted to narrowly taxonomic subjects, but should include the wider aspects of systematics and be integrated with evolutionary, cytological, genetical and physiological work. On the other hand, systematics without taxonomy will lead to an equally unbalanced point of view.

The specialized training of students in taxonomy must of necessity follow the undergraduate course. The kind and duration of the training will depend to a large extent on the future occupation which is envisaged for the candidates. One approach is to establish one-year M.Sc. or diploma courses in taxonomy, like those recently initiated at Edinburgh and Liverpool. These are directed at providing adequate preparation in taxonomic methods and procedures for those who are unable to spend 2-3 years on a $\mathrm{Ph} . \mathrm{D}$. course, or who do not require such extensive training (it should be remembered that a short taxonomic training may be required for people working in related fields). For the successful running of such 1-year training courses it will be necessary to establish postgraduate schools of taxonomy, each staffed by several taxonomists. Two or three universities should set up such schools as part of their forthcoming expansion plans. Wherever possible they should be based on existing nuclei in univorsities where the existing staff and facilities could be quickly increased. There is also much to be said for such a school to be formed at one of the new universities. The appointment of additional taxonomists scattered over the country is not a satisfactory alternative to the establishment of a few postgraduate schools of taxonomy, since lone taxonomists can no more be expected to make a satisfactory contribution to teaching and research in a large biological school than can any other lone scientists.

After a training course for one year in taxonomic methods, as suggested here, the best experience for a future taxonomist is a period of creative research leading to a Ph.D degree. This stage will require special herbarium, museum or library facilities. It is for this reason that the number of schools of taxonomy should be limited. Adequate provision for the financing of fieldwork, both at home and abroad, should be made, and this will be assisted by the proposal to set up "Wallace" studentships made in the Royal Society Report ${ }^{1}$. It is also essential that financial provision be made for travel to consult material in other institutions, in this country and abroad.

Research in taxonomy and systematics is closely linked with teaching and training in these fields. Thriving research schools cost money, and it should be the aim of the schools of taxonomy which are recommended here to provide facilities which are comparable with the best found in the United States and on the Continent. In some of the fields of systematics which are listed below as appropriate for further development, little satisfactory progress can be made if the basic resources are not available. There is a need for intimate practical knowledge of traditional approaches if radical new developments are to be expected. A large number of such developments of interest both to systematics and taxonomy are being initiated even with the limited support that is at present available. But there are many aspects of taxonomy which have remained essentially unchanged for decades or even centuries, and up-to-date methods are neglected. This is in part a consequence of the concentration of Britain's taxonomic resources in the national museums which are largely obliged-by their nature and finance- to restrict the amount of pure research they can carry out without prejudice to their heavy practical obligations. Their divorce from the universities and academic research institutions further aggravates this situation. It is urgent that, in addition to the build-up of basic resources and facilities in a few selected universities, substantial support should be given to setting up research units in such fields as comparative biochemistry, comparative physiology and ethology, comparative serology, data-processing methods and machines, developmental physiology, environmental control, epigenetics, population cytology, DNA comparison, numerical taxonomy, palæobiochemistry, protein-sequence taxonomy and theoretical taxonomy.

Systematics occupies to-day a dual role in biology. Systematists have the function of evaluating, interpreting and co-ordinating the greatly increased flow of data from many fields so as to serve biology as helpfully as possible. They are also having to assume, increasingly, responsibility for developing many of those aspects of biology that are discarded by its advancing spearheads. If other biologists wish systematists to occupy these roles effectively, they must be prepared to accept that adequate support be made available to them for teaching and research.

Taxonomy: Rep. Committee appointed by the Council of the Royal Society (Royai Society, London, 1963).

Nature, 198, 346 (1963).

\title{
B.B.C. ENGINEERING MONOGRAPHS
}

$\mathrm{S}^{\mathrm{E}}$ EVERAL times a year the Engineering Division of the British Broadcasting Corporation publishes a monograph either describing some novel technical equip. ment used in the development and operation of the Corporation's sound and television services, or making an appraisal of some aspect of its engineering activities. For example, Monograph No. 46 was published in February 1963, under the title The Application of Transistors to Sound Broadcasting, by S. D. Berry*. This describes

* British Broadcasting Corporation. BBC Engineering Monograph, No. 46 The Application of Transistors to Sound Broadcasting. By S. D. Berry
Pp. 20. (London: British Broadcasting Corporation, 1963.) 5s. the circuit arrangements and mechanical construction of a number of transistor audio-frequency amplifiers and associated apparatus designed for general use in the sound broadcasting services of the B.B.C.

Of a somewhat different type is Monograph No. 48 (June 1963) entitled The Development of B.B.C. Internal Telecommunications, by J. M. Chorley and J. S. Norwell †. In order to support the sound and television broadcasting services a comprehensive network of telecommunications

$\dagger$ British Broadcasting Corporation. BBC Engineering Monograph, No. 48 : and J. S. Norwell. Pp. 23. (London: British Broadcasting Corporation, 1963.) 58 . 
is essential. While the British Post Office provides many of the required facilities over its country-wide network, the Corporation is concerned with special circuits and, particularly, with their termination at various centres throughout the United Kingdom. This Monograph traces the development of such B.B.C. telecommunications and describes some of the equipment used. It does not, however, deal with the distribution of television and sound programmes.

The most recent of the published Monographs (No. 52: March 1964) is entitled Stereophony: The Effect of CrossTalk Between Left and Right Channels, by H. D. Harwood and D. E. L. Shortert. As the short list of references appended to this publication shows, the B.B.C. has been exploring the practical possibilities of stereophonic broadcasting for several years. One of the technical defects which may appear in a stereophonic sound system is the unintentional transfer of a portion of the signal in one channel to the other channel, a phenomenon

¥ British Broadcasting Corporation. BBC Engineering Monograph, No. 52 : Stereophony-The Effect of Cross-Talk Between Left and Right Channels. By H. D. Harwood and D. E. L. Shorter. Pp. 23. (London: JBritish Broadcasting Corporation, 1964.) 5 s. known as 'cross-talk' in telephone engineering. This results in a displacement or blurring of the reproduced sound images. The experiments described have covered the investigation of certain occurrences of cross-talk increasing at high or low frequencies, as well as crosstalk being independent of frequency. It was concluded that the minimum perceptible degree of cross-talk is influenced to some extent by the position of the observer in the listening room, but is largely independent of the acoustics of the room and of the bandwidth of the system.

Other Monographs published recently deal with such differing subjects as the measurement of the non-linear distortion generated by a loud-speaker system, and the methods of testing and measuring the properties of lenses used in the photoelectric components of television cameras. All these publications serve to demonstrate the extent to which the operation of the British Broadcasting Corporation's services are well supported by the comprehensive work of the Research, Designs and Lines Departments of the Corporation's Engineering Division.

R. L. Smith-Rose

\title{
ISOLATION OF SEROLOGICALLY ACTIVE FUCOSE-CONTAINING OLIGOSACCHARIDES FROM HUMAN BLOOD-GROUP H SUBSTANCE
}

\author{
By Mrs. VAIJAYANTI P. REGE, Dr. T. J. PAINTER, Dr. WINIFRED M. WATKINS \\ and Prof. W. T. J. MORGAN, C.B.E., F.R.S. \\ Lister Institute of Preventive Medicine, London, S.W.I
}

\begin{abstract}
$\mathrm{T}$ HE results of hæmagglutination inhibition experiments demonstrated more than ten years ago that L-fucose, or more exactly an $O-\alpha$-L-fucopyranosyl structure, is an important part of the serologically specific grouping in the $\mathrm{H}$-specific blood-group substance ${ }^{1}$. Since that time other evidence, involving the inhibition by $L$-fucose of the enzymatic decomposition of $H$ substance ${ }^{2}$ and the demonstration that L-fucose is the sugar most readily liberated from $H$ substance by the action of specific H-decomposing enzymes from Trichomonas foetus ${ }^{3}$ and from Bacillus fulminans ${ }^{4}$, has supported these early conclusions. More recently, results of a further serological analysis ${ }^{5}$ with the eel serum anti-H reagent suggested that the L-fucose concerned in $\mathrm{H}$ specificity may be joined to the next sugar by an $\alpha-(1 \rightarrow 2)$ linkage. Thus, $2-O-\alpha-\mathrm{L}$ -

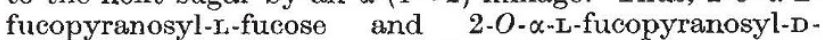
galactose are more powerful inhibitors than either 3-O- $\alpha$-L-fucopyranosyl-L-fucose or 4-O- $\alpha$-L-fucopyranosylL-fucose.

Difficulties in drawing clear-cut inferences from these results arise from the fact that methyl- $\alpha-L$-fucopyranosido inhibits the rel reagent as strongly as the disaccharides containing the $\alpha$-(1 $\rightarrow 2)$-linkages and that other oligosaccharides, such as lacto-N-fucopentaose I (ref. 6), are poor inhibitors of hæmagglutination although they contain $\alpha$-L-fucopyranosyl- $(1 \rightarrow 2)$-galactose units as terminal nonreducing end-groups. The anti-H reagent extracted from Lotus tetragonolobus seeds shows similar anomalies ${ }^{5}$, and it was pointed out that the failure to obtain greater inhibition with $\alpha-L$-fucosyl- $(I \rightarrow 2)$ compounds than with the simple methyl $\alpha$-L-fucoside might be due to the limitation imposed by the heterologous nature of the eel and plant seed anti-H agglutinins, the receptor sites of which may be largely saturated by a simple $\alpha$-L-fucopyranosyl grouping. Nevertheless, further support for the belief that $\mathrm{L}$-fucose is linked $\alpha-(l \rightarrow 2)$ in the $H$ determinant structure was gained from experiments with partially purified enzymes from $T$. foetus $^{7}$; preparations which destroy the serological activity
\end{abstract}

of $\mathrm{H}$ substance split fucose from oligosaccharides in which the sugar is joined by $\alpha-(1 \rightarrow 2)$ linkage to galactose but not from an oligosaccharide in which $L$-fucose is joined by a $(1 \rightarrow 4)$ linkage to $N$-acetyl-D-glucosamine.

That more than one terminal non-reducing sugar residue may be important for $\mathrm{H}$ specificity was indicated by inhibition experiments with anti-H reagents from seeds of the plants Cytisus sessilifolius and Laburnum alpinum These reagents are strongly inhibited by human $\mathbf{H}$ substances but are not inhibited by L-fucose ${ }^{8}$. The disaccharido 4- $O$ - ( $N$ - acetyl - $\beta$-D - glucosaminopyranosyl $)-N$ - acetyl - D glucosamine ( $N, N^{\prime}$-diacetyl-chitobiose), however, inhibits both Cytisus and Laburnum reagents ${ }^{8}$, indicating that an $N$-acetyl- $\beta$-D-glucosaminopyranosyl structure may be a serological determinant unit in $\mathrm{H}$-substance in addition to an $\alpha$-L-fucopyranosyl residue.

Valuable as the results of inhibition and enzymatic experiments are as indirect pointers to the structures of the serologically active groupings in the blood-group macromolecules, the final proof of structure must rest on the chemical characterization of active fragments isolated from the substances themselves. Examination of the four di- and the three tri-saccharides obtained from the partial acid hydrolysis products of a human blood-group $H$ specific substance ${ }^{8,9}$ revealed two serologically active fragments, the disaccharide 3-O- $(N$-acetyl- $\beta$-D-glucosaminopyranosyl $)-\mathrm{D}$-galactose, and the trisaccharide $\mathrm{O}-(\mathrm{N}$ acetyl- $\beta$ - D - glucosaminopyranosyl $)$ - $(1 \rightarrow 3)$ - $O-\beta-\mathrm{D}$ - galactopyranosyl- $(1 \rightarrow 3)-N$-acetyl-D-galactosamine. These compounds, however, inhibited only the Cytisus anti-H reagent and failed to inhibit human, rabbit or eel anti-H agglutinins. This is not surprising, as none of the oligosaccharides described contained L-fucose owing to the rapid liberation of this sugar under the conditions of hydrolysis used. An investigation of the isolated fragments indicated the presence of two main types of carbohydrate chain in $\mathrm{H}^{\prime}$ substance for which the following partial structures were proposed $^{9}$ : 